\title{
A GENERALISATION OF THE STIELTJES TRANSFORM
}

\section{by A. K. CHATTERJEE}

(Received 31st January 1963)

1. Transforms of the type

$$
f(p)=\int_{0}^{\infty} F(t, p) \phi(t) d t
$$

have long been known. If we take

$$
F(t, p)=(t+p)^{-\sigma} e^{\frac{t}{2}(t+p)} W_{k, \mu}(t+p),
$$

where $W_{k, \mu}(x)$ denotes the Whittaker function, we obtain the following transform:

$$
f(p)=\int_{0}^{\infty}(t+p)^{-\sigma} e^{\frac{t}{2}(t+p)} W_{k, \mu}(t+p) \phi(t) d t .
$$

When $k+\mu=\frac{1}{2}$ and $k-\sigma=-1$,

$$
F(t, p)=\frac{1}{t+p}
$$

and (1.3) reduces to the Stieltjes transform

$$
f(p)=\int_{0}^{\infty} \frac{1}{t+p} \phi(t) d t .
$$

We shall denote (1.3) symbolically as

2. Inversion formula Let

$$
f(p) \underset{k, \mu}{\stackrel{\sigma}{\longrightarrow}} \phi(t) .
$$

$$
\phi(t)=\left\{\begin{array}{l}
0\left(t^{\eta_{1}}\right), R\left(\eta_{1}\right)>0 \text { for small } t \\
0\left(t^{\eta_{2}}\right), R\left(\eta_{2}\right)<0 \text { for large } t
\end{array}\right.
$$

where $|\arg t|<\pi$ and $\phi(t)$ is of bounded variation in $(0, \infty)$.

Multiplying by $p^{\rho-1}$ and then integrating from 0 to $\infty$ with respect to $p$ and assuming that $\psi(\rho)$, the Mellin transform of $f(p)$, exists, we get

$$
\begin{aligned}
\int_{0}^{\infty} f(p) p^{\rho-1} d p & =\psi(\rho), \\
& =\int_{0}^{\infty} p^{\rho-1} d p \int_{0}^{\infty}(t+p)^{-\sigma} e^{\frac{1}{2}(t+p)} W_{k, \mu}(t+p) \phi(t) d t, \\
& =\int_{0}^{\infty} \phi(t) d t \int_{0}^{\infty} p^{\rho-1}(t+p)^{-\sigma} e^{\frac{1}{t}(t+p)} W_{k, \mu}(t+p) d t,
\end{aligned}
$$

E.M.S. - S 
on changing the order of integration. Now, by (1), p. 412 (51),

$$
\begin{aligned}
\int_{0}^{\infty} x^{\rho-1}(a+x)^{-\rho} e^{\frac{1}{2} x} W_{k, \mu}(a+x) d x= & \frac{\Gamma(\rho) a^{\rho} e^{-\frac{1}{2} a}}{\Gamma\left(\frac{1}{2}-k+\mu\right) \Gamma\left(\frac{1}{2}-k-\mu\right)} \\
& \times G_{23}^{31}\left(a \mid \begin{array}{l}
k-\sigma+1,0 \\
-e, \frac{1}{2}+\mu-\sigma, \frac{1}{2}-\mu-\sigma
\end{array}\right),
\end{aligned}
$$

where $|\arg a|<\pi, 0<R(\rho)<R(\sigma-k)$. Hence we get

$\psi(\rho)=\frac{\Gamma(\rho)}{\Gamma\left(\frac{1}{2}-k+\mu\right) \Gamma\left(\frac{1}{2}-k-\mu\right)} \int_{0}^{\infty} t^{\rho} \phi(t) G_{23}^{31}\left(t \mid \begin{array}{l}t-\sigma+1,0 \\ -\rho, \frac{1}{2}+\mu-\sigma, \frac{1}{2}-\mu-\sigma\end{array}\right) d t$,

where $|\arg t|<\pi, 0<R(\rho)<R(\sigma-k)$.

Now, applying Mellin's inversion formula, we get

$$
\phi(t)=\frac{1}{2 \pi i} \int_{c-i \infty}^{c+i \infty} \psi(\rho) t^{-\rho} d \rho
$$

\section{Uniqueness theorem}

Let $\phi_{1}$ and $\phi_{2}$ be continuous in $t \geqq 0$ and

$$
f(p) \underset{k, \mu}{\stackrel{\sigma}{\longrightarrow}} \phi_{1}(t)
$$

and also

$$
f(p) \underset{k, \mu}{\stackrel{\sigma}{\longrightarrow}} \phi_{2}(t) \text {. }
$$

Then

$$
\phi_{1}(t) \equiv \phi_{2}(t)
$$

In future work the equation (2.2) will be called the SA-transform of $\phi(t)$.

The author is grateful to Prof. Brij Mohan for his help and guidance in the preparation of this note.

\section{REFERENCE}

(1) H. Bateman, Tables of integral transforms, Vol. II, 411-412.

\section{DePartment of MATHEMATICS}

VARANASI-5, INDIA 